\title{
Contents Index to Volume 2
}

\section{Perspectives}

Smart Data for Digital Humanities

Marcia Lei Zeng

2017,2(1), 1-12

Big Data and Data Science: Opportunities and Challenges of iSchools

II-Yeol Song \& Yongjun Zhu

Vol. 2 No. 4, 2017

pp $95-96$

2017,2(3),1-18 DOI: 10.1515/jdis-2017-0022

Understanding Big Data for Industrial Innovation and Design: The Missing Information Systems Perspective

Miguel Baptista Nunes

2017,2(4),1-6

A Framework for the Assessment of Research and Its Impacts

Cinzia Daraio

$2017,2(4), 7-42$

\section{Expert Reviews}

Patent Citations Analysis and Its Value in Research Evaluation: A Review and a New Approach to Map Technology-relevant Research

Anthony F.J. van Raan

$2017,2(1), 13-50$

Science Mapping: A Systematic Review of the Literature

Chaomei Chen

2017,2(2), $1-40$

Big Metadata, Smart Metadata, and Metadata Capital: Toward Greater Synergy Between Data Science and Metadata

Jane Greenberg

2017,2(3),19-36

Rediscovering Don Swanson: The Past, Present and Future of Literature-based Discovery

Neil R. Smalheiser

2017,2(4),43-64

\section{Research Papers}

Functions of Uni- and Multi-citations: Implications for Weighted Citation Analysis

Dangzhi Zhao, Alicia Cappello \& Lucinda Johnston

2017,2(1),51-69

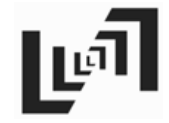

Insight into the Disciplinary Structure of Nanoscience \& Nanotechnology

Chunjuan Luan \& Alan L. Porter

2017,2(1), 70-88

Usage Count: A New Indicator to Detect Research Fronts 


\section{Contents Index}

Provenance Description of Metadata Vocabularies for the Long-term Maintenance of Metadata

Chunqiu Li \& Shigeo Sugimoto

Enhancing Navigability: An Algorithm for Constructing Tag Trees

Chong Chen \& Pengcheng Luo

Seeking Health Information Online: The Moderating Effects of Problematic Situations on User Intention

Lidan Xia, Shengli Deng \& Yirong Liu

Detecting Dynamics of Hot Topics with Alluvial Diagrams: A Timeline Visualization

Wenjing Ruan, Haiyan Hou \& Zhigang Hu

Digitizing Dunhuang Cultural Heritage: A User Evaluation of Mogao Cave Panorama Digital Library

Xiao Hu, Eric M. Y. Ho \& Chen Qiao

Visualization of Disciplinary Profiles: Enhanced Science Overlay Maps

Stephen Carley, Alan L. Porter, Ismael Rafols \& Loet Leydesdorff

An Accurate and Impartial Expert Assignment Method for Scientific Project Review

Mingliang Yue, Kailin Tian \& Tingcan Ma

Using Machine Reading to Understand Alzheimer's and Related Diseases from the Literature

Satoshi Tsutsui, Yi Bu \& Ying Ding 